\title{
Tratamento odontológico em portador da Doença de Huntington: relato de caso
}

Dental treatment in Huntington's Disease: a case report

Tratamiento dental en la Enfermedad de Huntington: reporte de un caso

\section{Regina Maria RAFFAELE ${ }^{1}$}

Mario Eduardo BALDO ${ }^{1}$

Renata Lanzonni de OLIVEIRA ${ }^{2}$

Paulo Pereira do NASCIMENTO2

Paulo Roberto Haidamus de Oliveira BASTOS ${ }^{2}$

Ellen Cristina GAETTI JARDIM ${ }^{2}$

${ }^{1}$ Associação Beneficente Santa Casa de Campo Grande - Campo Grande - MS, Brasil

${ }^{2}$ Universidade Federal de Mato Grosso do Sul - Campo Grande - MS, Brasil

\section{Resumo}

Caracterizada por um distúrbio neurodegenerativo progressivo autossômico dominante, a doença de Huntington interfere na capacidade de movimento, interação cognitiva além de causar trantornos comportamentais nos individuos diagnosticados com a enfermidade. Dependendo do estadiamento e do grau de comprometimento neuromotor a adesão voluntária à propostas terapeuticas como o tratamento odontológico fica comprometida. Este estudo relata o caso raro de um portador da doença de Huntington do sexo masculino com 23 anos, diagnosticado com a doença desde 2017. A abordagem em âmbito hospitalar sob sedação foi adotada considerando as limitações físicas e segurança para o paciente bem como a complexidade e extensão dos procedimentos odontológicos necessários.

Descritores: Doença de Huntington; Odontologia Hospitalar; Tratamento Odontológico.

\section{Abstract}

Characterized by a progressive autosomal dominant neurodegenerative disorder, Huntington's disease interferes in the ability to move, cognitive interaction and causes behavioral disorders in individuals diagnosed with the disease. Depending on the staging and the degree of neuromotor damage, willingly adhesion to therapeutic procedures such as a dental treatment could be compromised. This study reports the rare case of a 23-year-old male with Huntington's disease diagnosed since 2017. A hospital approach under sedation was required considering the physical limitations and safety for the patient as well as the complexity and extension of the necessary dental procedures.

Descriptors: Huntington Disease; Hospital Odontology; Dental Treatment.

\section{Resumen}

Caracterizada por un trastorno neurodegenerativo progresivo autosómico dominante, la enfermedad de Huntington interfiere con la capacidad para moverse, la interacción cognitiva y causa trastornos del comportamiento en individuos diagnosticados con la enfermedad. Según la estadificación y el grado de afectación neuromotora, se compromete la adherencia voluntaria a propuestas terapéuticas como el tratamiento odontológico. Este estudio reporta el caso raro de un varón de 23 años con enfermedad de Huntington diagnosticado con la enfermedad desde 2017. Se adoptó el abordaje hospitalario bajo sedación considerando las limitaciones físicas y la seguridad del paciente, así como la complejidad y extensión de los procedimientos dentales necesarios.

Descriptores: Enfermedad de Huntington; Odontología Hospitalaria; Tratamiento Dental.

\section{INTRODUÇÃO}

A doença de Huntington é distúrbio neurodegenerativo progressivo raro, de caráter autossômico dominante. É mais prevalente em homens, tendo seu início relativamente tardio, de 30 a 45 anos de idade, podendo haver sintomas mais precoces ${ }^{1,2}$.

É caracterizado por distúrbio de movimento, declínio cognitivo e sintomas comportamentais ${ }^{3,4}$. Também podem apresentar diminuição da percepção da dor, podendo estar relacionada às áreas cerebrais afetadas pela doença $a^{5,6}$.

Seu diagnóstico é feito com base na história familiar, testes genéticos para expansão CAG em HTT e avaliação clínica. Os tratamentos são limitados às terapias para tratar os sintomas com auxílio de uma equipe multiprofissional. $\mathrm{Na}$ odontologia se torna um desafio 0 tratamento de pacientes com distúrbios cerebrais neurodegenerativos. Em conjunto com os médicos neurologista e anestesiologista, o tratamento odontológico sob anestesia geral pode ser uma melhor opção, fornecendo proteção das vias aéreas e uma recuperação rápida e segura para os acometidos pela enfermidade ${ }^{7,8}$.
CASO CLÍNICO

Deste modo, paciente do sexo masculino, 23 anos, com diagnóstico de doença de Huntington desde 2017, obtido por exame molecular, tendo o pai o mesmo diagnóstico. Apresenta movimentos involuntários, debilidade motora e dificuldade de marcha. Compareceu ao consultório odontológico acompanhado pela mãe, com queixa de dificuldade coordenação motora para realizar escovação dentária eficaz e destruição coronária em vários dentes, porém sem sintomatologia dolorosa. Ao exame clínico observou-se a doença cárie em estágio avançado em vinte e cinco dentes e sete raízes residuais.

Devido à condição física do paciente, não seria possível realizar todos os procedimentos em ambulatório odontológico. Com isso, optou-se pelo tratamento em âmbito hospitalar sob anestesia geral em centro cirúrgico para melhor conforto e segurança do paciente. Foram realizadas as restaurações definitivas em resina composta dos elementos dentários cariados e exodontias das raízes residuais e remoção dos dentes inclusos. Todo o tratamento teve duração de 8 horas, sem intercorrências. Em seguida, os familiares foram 
orientados a procurar uma profissional terapeuta ocupacional para poder desenvolver um dispositivo acoplado à escova dental para melhor manuseio do paciente. O paciente é monitorado pelo ambulatório da equipe de odontologia periodicamente visando a manutenção da saúde periodontal e controle de placa bacteriana (Figuras 1 a 3 ).

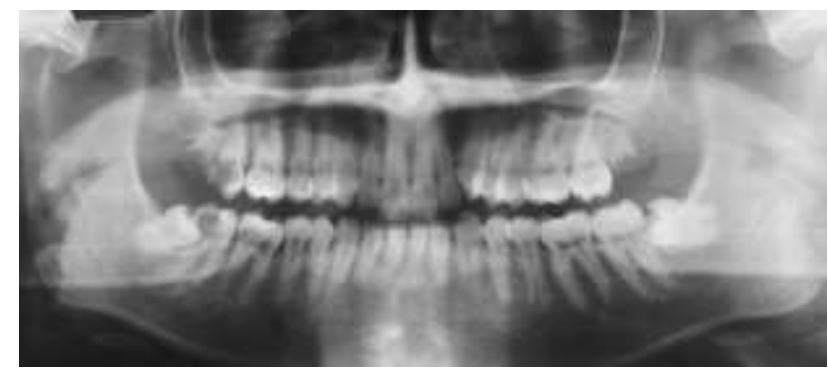

Figura 1: Imagem da radiografia panorâmica evidenciando lesões de cárie, destruição coronária e dentes inclusos.

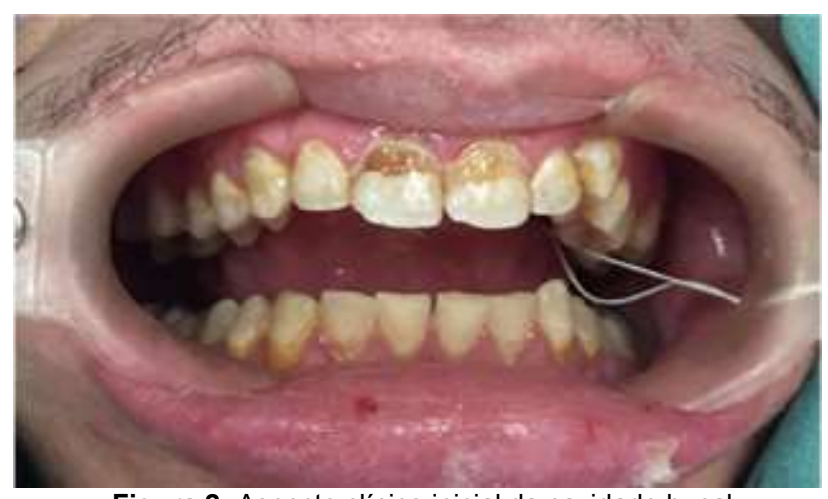

Figura 2: Aspecto clínico inicial da cavidade bucal.

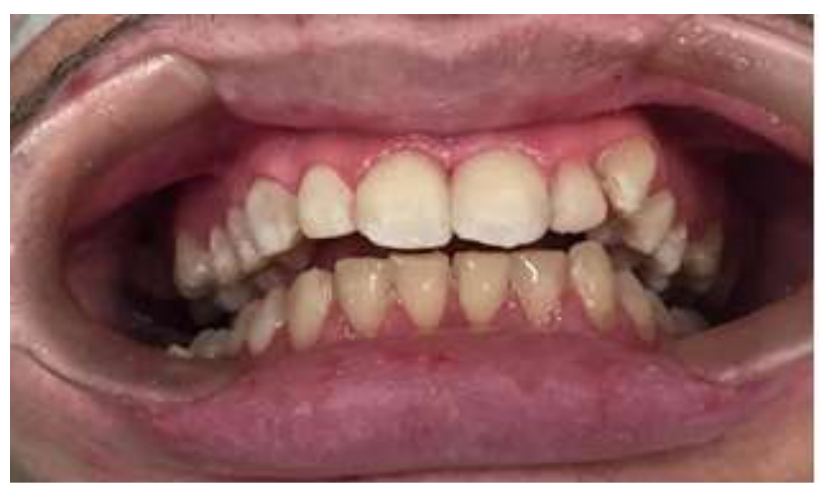

Figura 3: Aspecto clínico final após realizado as restaurações permanentes e exodontias.

DISCUSSÃO

Doenças degenerativas que acometem o sistema nervoso geralmente são responsáveis por favorecer agravos de saúde bucal em seus portadores, seja limitando os movimentos ${ }^{4}$ durante a higiene oral ou mesmo interferindo na percepção de dor nestes pacientes ${ }^{6}$, o que por si só já incorreria em sérias complicações odontológicas levando em consideração que a dor corresponde a um importante sinalizador da progressão tanto da cárie quanto da doença periodontal. Por outro lado, a dificuldade de marcha e locomoção ${ }^{3,4}$, acrescidas de movimentos espasmódicos involuntários reduzem significativamente as chances de sucesso do tratamento odontológico pela via ambulatorial, assim sendo a busca por propostas alternativas de intervenção nesses casos quase sempre leva a uma abordagem multidisciplinar realizada em âmbito hospitalar ${ }^{7}$.

A realização do tratamento odontológico no centro cirúrgico, além de proporcionar maior segurança para o profissional, considerando que este atua amparado pela equipe médica ${ }^{8}$, dada a quantidade de procedimentos cruentos necessários neste caso mostrou-se benéfica para o paciente, especialmente se considerarmos a remoção quase que imediata dos focos de infecção e interrupção da progressão das lesões de cárie às custas de uma única intervenção.

Em virtude do exposto, o diagnóstico precoce da doença cárie é imprescindível para evitar a destruição coronária e perda de elementos dentários. A falta de sintomatologia, somado a dificuldade motora ${ }^{9}$ e uma dieta cariogênica podem ser fatores relevantes para o aceleramento desta situação. Estes focos de infecção podem levar a quadros sistêmicos mais graves, como pneumonia e endocardite.

CONCLUSÃO

A presença do cirurgião-dentista atuando no âmbito hospitalar, em centro cirúrgico, favorece significativamente na remoção dos focos infeciosos de origem bucal impedindo a evolução destes como comorbidades aos portadores da Doença de Huntington além de contribuir para o bem-estar e autoestima desses pacientes.

\section{REFERÊNCIAS}

1. MacPherson P, Harper I, MacDonald I. Propofol and remifentanil total intravenous anesthesia for a patient with Huntington disease. J Clin Anesth. 2004;16:537-8.3.

2. Gilli E, Bartoloni A, Fiocca F, Dall'Antonia F, Carluccio $S$. Anaesthetic management in a case of Huntington's chorea. Minerva Anestesiol. 2006;72:757-62.

3. Quarrell OW, Nance MA, Nopoulos P, Paulsen JS, Smith JA, Squitieri F. Managing juvenile Huntington's disease. Neurodegener. Dis Manag. 2013; 3(3):267-76.

4. Baig SS, Strong M, Quarrell OW. The global prevalence of Huntington's disease: a systematic review and discussion. Neurodegener. Dis Manag. 2016;6(4):331-43.

5. Perrotta A, Serpino C, Cormio C, Serrao $M$, Sandrini G, Pierelli $F$ et al. Abnormal spinal cord pain processing in Huntington's disease. The role of the diffuse noxious inhibitory control. Clin Neurophysiol. 2012;123:1624-630.

6. Sprenger $G$, van der Zwaan $K$, Roos R, Achterberg $\mathrm{W}$. The prevalence and the burden 
of pain in patients with Huntington disease a systematic review and meta-analysis. Pain.2019;160(4):773-83.

7. Rada RE. Comprehensive Dental Treatment of a Patient with Huntington's disease: literature review and case report. Spec Care Dentist. 2008;28(4):131- 35.

8. Haimov-Kaldess I,Haim D,Garfunkel A.General Anesthesia for Dental Treatment in a Patient With Huntington's Chorea. Compend Contin Educ Dent. 2016;37(1):22-5.

9. Martelli A. Aspectos clínicos e fisiopatológicos da Doença de Huntington. Arch Health Invest. 2014;3(4):32-39.

\section{CONFLITO DE INTERESSES}

Os autores declaram não haver conflitos de interesse

AUTOR PARA CORRESPONDÊNCIA

\section{Regina Maria Raffaele}

Chefe da Divisão Clínica do Serviço de Odontologia Hospitalar da Associação Beneficente de Campo Grande Santa Casa

Telefone (67) 992975939

E-mail: reginaraffaele@hotmail.com 